\title{
Camera-Augmented Mobile C-arm (CAMC) Application: 3D Reconstruction Using a Low-Cost Mobile C-arm
}

\author{
N. Navab ${ }^{1}$, M. Mitschke ${ }^{2}$, and O. Schütz ${ }^{2}$ \\ 1 Siemens Corporate Research \\ 755 College Road East, Princeton, NJ 08540, USA \\ 2 Siemens AG, Medical Engineering \\ Henkestr. 127, 91052 Erlangen, Germany \\ Nassir.Navab@scr.siemens.com \\ $\{$ Matthias.Mitschke, Oliver.Schuetz\}@med.siemens.de
}

\begin{abstract}
High-end X-ray C-arm gantries have recently been used for $3 \mathrm{D}$ reconstruction. Low-cost mobile C-arms enjoy the advantage of being readily available and are often used as interventional imaging device, but do not guarantee the reproducibility of their motion. The calibration and reconstruction process used for high-end $\mathrm{C}$-arms cannot be applied to them. Camera-Augmented Mobile C-arm (CAMC) is the solution we propose. A CCD camera is attached to the (motorized) mobile $\mathrm{C}$-arm in order to calibrate the C-arm's projection geometry on-line. The relationship between X-ray and camera projection geometry is characterized in an off-line calibration process. We propose the notion of Virtual Detector (VD), which enables us to describe both optical and X-ray geometry as pinhole cameras with fixed intrinsic parameters. We have conducted experiments in order to compare the results of CAMC calibration with the calibration method used for high-end C-arms and using an optical tracking system (Polaris from Northern Digital, Inc.).
\end{abstract}

\section{Introduction}

C-arm X-ray systems are used in many surgical procedures such as fluoroscopybased orthopedic procedures. Even if a 3D CT reconstruction is computed offline, often X-ray fluoroscopy is used as interventional image-guided modality. However, the success of the procedure depends on the ability of the surgeon to mentally recreate the spatio-temporal intraoperative situation from twodimensional fluoroscopic X-ray images. In this work we would like to provide $3 \mathrm{D}$ reconstruction using the mobile $\mathrm{C}$-arm itself. This eliminates the need for a pre-computed CT reconstruction. The same mobile $\mathrm{C}$-arm is also used for intraoperative guidance. The registration between the $3 \mathrm{D}$ data and $2 \mathrm{D}$ fluoroscopic images is much easier since they are obtained with the same modality.

The major reason that mobile C-arm systems are still not used for 3D reconstruction is that, in general, they do not guarantee reproducible motion for consecutive examinations. The solution we propose to this problem is to compute 


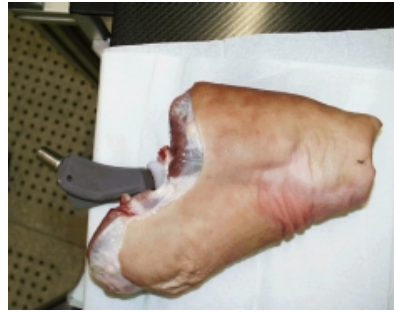

original optical image

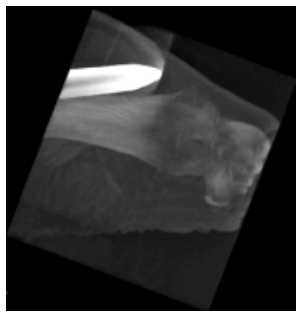

$3 \mathrm{D}$ reconstruction

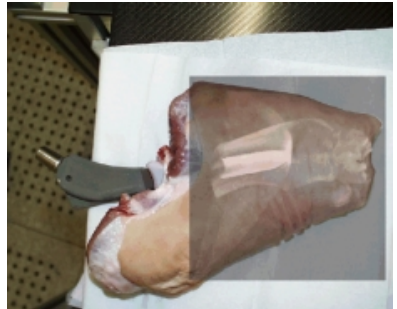

result of overlay

Fig. 1. CAMC could enable the superimposition of X-ray on top of optical images

the calibration data during the patient run. Markers opaque to X-ray which are used in general for off-line calibration of C-arms cannot be used on-line, because they may interfere with the region of interest. Therefore we use optical markers which are transparent to X-ray in order to compute the motion of the mobile $\mathrm{C}$-arm with the attached CCD camera. In this paper we present first results of 3D reconstruction using a Camera-Augmented Mobile C-arm (CAMC). This is only one of the possible advantages of using CCDs attached to a mobile C-arm. CAMC can also provide new possibilities for tracking, navigation and augmented reality. The optical camera can be used for registration between patient's optical and X-ray images. This would enable the system to combine X-ray and optical images and get images similar to Fig. 1. The "enhanced reality display" [5] and image overlay system for medical imaging [1] present excellent techniques for combining patient images with different medical imaging data. In both cases external sensors are brought in the operating room, and complicated registration and tracking procedures are necessary. The advantage of CAMC is that the registration is much easier since optical and X-ray system are rigidly attached. If equipped with a camera, the mobile $\mathrm{C}$-arm which is often readily available in medical environment will be able to provide X-ray images, 3D reconstructed data, optical images and finally combined X-ray/optical images.

In this paper we present our first steps towards building such a CameraAugmented Mobile C-arm system. We define the relationship between X-ray and CCD imaging geometry and use the attached CCD camera to characterize the motion of $\mathrm{C}$-arm. We then present our first 3D reconstruction results using a mobile $\mathrm{C}$-arm. We compare this result with the one obtained using $\mathrm{X}$-ray phantoms (this would be ideal but it is not possible in real-time application) and using an external tracking sensor. 


\section{3D Reconstruction from Mobile C-arm ${ }^{1}$}

In order to obtain the three-dimensional reconstruction the position of the $\mathrm{C}$-arm mounted X-ray source and detector relative to the patient has to be measured. If the $\mathrm{C}$-arm geometry is reproducible, the geometrical calibration can be done off-line using X-ray phantoms $[9,2,7]$ and the pose information can be used for the patient examination. However mobile C-arm systems do not guarantee reproducible motion for consecutive examinations.

Here, we propose to use a CCD camera attached to the C-arm in order to estimate the $\mathrm{C}$-arm motion parameters and recover the $\mathrm{X}$-ray projection geometry on-line. However the detector plane may also move relative to the X-ray source during the patient run. Therefore, we use a set of X-ray markers positioned on a plate directly in front of the X-ray source. These markers are designed and positioned in order to cover a small border of the X-ray image. In theory one can define a set of planar transformations that can be applied to the X-ray images before back-projection without affecting the $3 \mathrm{D}$ reconstruction results. We use the image of the marker plate to warp the X-ray image onto a virtual plane, we call the "virtual detector plane", before back-projection. By fixing this virtual plane, i.e. warping all X-ray images taken during the patient run such that the marker plate appears as it does on the virtual detector plane, one can fix the $\mathrm{X}$-ray projection geometry relative to X-ray source. We then combine this information with $\mathrm{C}$-arm motion computed using a CCD camera attached to the $\mathrm{X}$-ray source in order to compute the full X-ray projection geometry during the patient run. This procedure provides a set of projection matrices, which can be used in order to back-project the X-ray image onto the voxel space for the $3 \mathrm{D}$ reconstruction process (see references for more details on back-projection and $3 \mathrm{D}$ reconstruction procedures $[8,3])$.

\subsection{X-ray and Camera Projection Geometry}

The X-ray projection geometry as well as optical camera geometry is represented by $\mathbf{P}$ a $3 \times 4$ homogeneous matrix of projection. This matrix represents all the imaging geometry parameters which can be divided into two sets. The first set is called the extrinsic parameters. They define position and orientation of the imaging system in a world coordinate system. The second set is called the intrinsic parameters. They only depend on internal parameters of the imaging system. For the optical camera the intrinsic parameters could be defined as pixel size, image center, and principle distance or focal length. For a radiographic imaging system the intrinsic parameters could be defined as pixel size, image center, and source to detector distance. Note that the last two parameters are functions of relative position and orientation of source and detector. Both Xray and optical imaging systems are modeled after a simple pinhole camera. A C-arm coordinate system is defined with its origin at the X-ray source. An

\footnotetext{
${ }^{1}$ Here, we consider mobile C-arms equipped with a Solid State Detector with no distortion artifact. Distortion correction is necessary for traditional systems $[4,6]$
} 
optical camera coordinate system is defined with its origin at the optical center. We define the z-axis parallel to the normal dropping from X-ray source or the optical center for an optical camera onto the image plane. The $\mathrm{x}$-axis and $\mathrm{y}$ axis are parallel to the row and column vectors of the CCD for camera and 2D detector plane for X-ray imaging system.

\subsection{Virtual Detector: Connection between X-ray and Optical Camera Projection Models}

For a CCD camera with fixed focal length it is reasonable to suppose that the intrinsic parameters remain fixed while acquiring a sequence of images. For Xray images this is not the case. The intrinsic parameters of our C-arm imaging system are not constant over the image series. This is due to minor torsion of the C-arm caused by the weights of both X-ray source and detector. In order to be able to take the motion estimated from a CCD camera and apply it to $\mathrm{X}$-ray geometry we need to fix the X-ray imaging intrinsic parameters. This is accomplished by a plate with a small number of X-ray opaque markers that is attached to the X-ray focus. These markers are projected onto the X-ray image close to the image borders. This bordering area is removed before the filtering and $3 \mathrm{D}$ reconstruction process. The marker plate is placed at a fixed position relative to the X-ray source. Therefore, its projection onto the image plane only depends on the intrinsic parameters of our X-ray imaging system.

Let us take two arbitrary X-ray images, $I$ and $I^{\prime}$. These two images correspond to two $\mathrm{X}$-ray projection matrices $\mathbf{P}$ and $\mathbf{P}^{\prime}$. We can define a $2 \mathrm{D}-2 \mathrm{D}$ transformation $\mathbf{H}$ that maps the positions of each marker, $\mathbf{m}_{i=1 \ldots k}(k=$ number of markers), on one X-ray image to its corresponding position on the other one, $\mathbf{m}_{i}^{\prime}$.

This planar transformation would actually warp one X-ray image onto an image plane at exactly same position and orientation relative to the $\mathrm{X}$-ray source as the other X-ray image (detector) plane. We have:

$$
\mathbf{m}_{i}^{\prime}=\mathbf{H m}_{i}, \quad i=1 \ldots k
$$

Instead of applying the transformation $\mathbf{H}$ to the image, we can simply multiply this transformation with the projection matrix $\mathbf{P}$. Thereby we obtain a projection matrix $\tilde{\mathbf{P}}$ with the extrinsic parameters of $\mathbf{P}, \mathrm{C}$-arm in the first position, and intrinsic parameters of $\mathbf{P}^{\prime}$, relative position of $\mathrm{X}$-ray source and detector plane, in the second position.

In this way, we take one of the X-ray images as a reference for fixing the relative position and orientation of X-ray source and detector plane, defining the virtual detector, and therefore fixing the intrinsic parameters, see Fig. 2. Now, we only need to compute the motion of the X-ray source, using the attached CCD camera, in order to recover all projection matrices.

\subsection{Determination of X-ray Projection Matrices for a CAMC}

Using the virtual detector described above allows us to treat our C-arm system as a pinhole camera with fixed intrinsic parameters. The motion of this $\mathrm{C}$-arm 

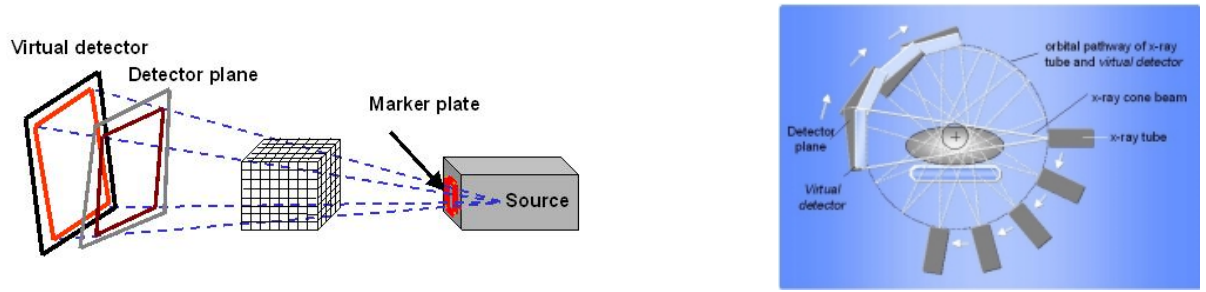

Fig. 2. Virtual Detector: The X-ray image can be first projected or backprojected onto a secondary plane, following the same X-ray projection geometry, and then back-projected onto the 3D voxel planes. The resulting 3D-2D mapping will theoretically remain unchanged (left). The intrinsic geometry of the C-arm remains unchanged for the whole patient examination when using the virtual detector (right).

imaging system (X-ray source with the virtual detector plane) for a whole image series will then be the same as the motion of a CCD camera attached to the X-ray source. Direct motion estimation from consecutive projection matrices as shown in [7] is more precise than motion estimation by decomposing these projection matrices and computing the extrinsic parameters for each frame. We therefore propose a scenario in which we do not need to compute the intrinsic parameters of either our X-ray imaging system or the optical one. The scenario is divided into two steps:

1. Off-line calibration: One pair of images (X-ray and optical) is taken. The two projection matrices $\mathbf{P}_{x}$ (X-ray) and $\mathbf{P}_{c}$ (CCD camera) are used as reference for the transformation between X-ray and CCD camera coordinate systems. The image of the marker plate markers on the X-ray image defines the virtual detector for the on-line calibration.

2. On-line calibration: For each frame, the motion of the X-ray source relative to its position in the off-line calibration step is computed using optical cameras. This is done by computing the projection matrix for optical camera and estimating the motion without ever computing its intrinsic parameters: $\left([\mathbf{R}, \mathbf{t}]=f\left(\mathbf{P}_{c}, \mathbf{P}_{c}^{\prime}\right)\right)$, see [7] for more details on direct motion estimation. We then apply this motion to the reference X-ray projection matrix $\mathbf{P}_{x}$, resulting in X-ray projection matrix $\tilde{\mathbf{P}}_{x}^{\prime}=\mathbf{P}_{x} \cdot\left[\begin{array}{cc}\mathbf{R} & \mathbf{t} \\ \mathbf{0}^{T} & 1\end{array}\right]$. This provides us with an X-ray projection matrix that projects $3 \mathrm{D}$ voxels onto our virtual detector plane for each frame. Using the marker plate (attached to the X-ray source) the planar transformation (2D-2D mapping) between the X-ray image and virtual detector $\mathbf{H}$ is computed (see sect. 2.2 for more details). The 3D-2D projection followed by the $2 \mathrm{D}-2 \mathrm{D}$ warping results in a new $3 \mathrm{D}-2 \mathrm{D}$ projection matrix $\mathbf{P}_{x}^{\prime}=\mathbf{H} \cdot \tilde{\mathbf{P}}_{x}^{\prime}$. This projection matrix, for each frame, provides the final mapping between $3 \mathrm{D}$ voxels and $2 \mathrm{D}$ pixels on the corresponding X-ray image. 

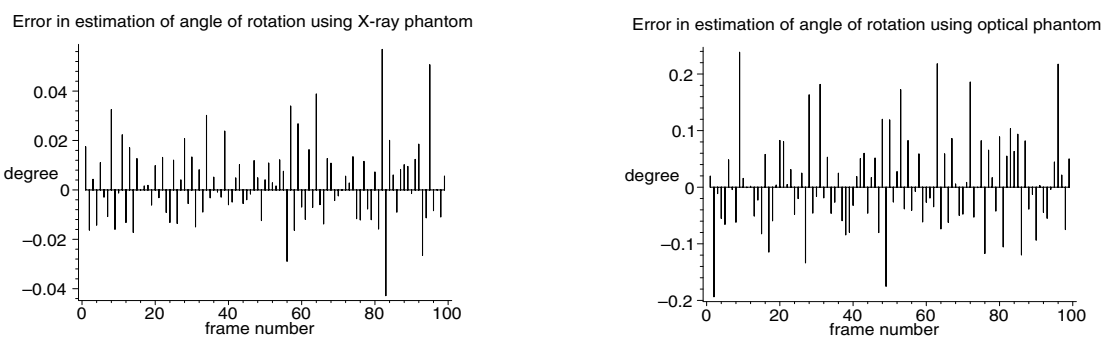

Fig. 3. Error in estimation of angle of rotation (in degrees) using X-ray (left) and optical (right) projection matrices versus ground truth of motion platform

These projection matrices are used for 3D reconstruction from an unstable mobile C-arm. The results are described in the following section.

\section{Experimental Results}

This section describes experiments conducted in order to evaluate the performance of the proposed method. For the first experiment we use a motion platform with precise ground truth information. In this case, we evaluate the calibration result using both X-ray and optical phantoms with regard to the achieved accuracy of estimated rotation. Then a second experiment is conducted using a mobile C-arm with no available ground truth. The results of $3 \mathrm{D}$ reconstruction will be used for a qualitative evaluation. Questions we try to answer are: Can pose and motion of the C-arm be estimated using a CCD camera ? Is the achieved accuracy good enough for 3D reconstruction?

\subsection{Motion Platform}

The goal of this first experiment is to quantitatively compare the accuracy of pose and motion estimation using optical and X-ray phantoms. We use the Xray phantom and calibration process used in [7]. X-ray phantoms interfere with the patient's X-ray image and cannot be used for mobile C-arm systems in real application. However, this gives us the advantage of comparing our results with $3 \mathrm{D}$ reconstruction results which have been proved by physicians and successfully tested on patients already.

We first use a precise motion platform, providing the ground truth, for a comparative study of motion estimations using X-ray and CCD phantoms. The position and orientation of X-ray and optical phantom as well as the motion platform is known in the same coordinate system. We use a composite phantom which has two distinct but connected parts. One part is the X-ray ring phantom (see [7]), the other part is an optical phantom.

We take sets of 100 pairs of simultaneous X-ray and optical images. The motion consists of 99 steps of 1.8 degrees pure rotation around a fixed axis. For all consecutive pairs of X-ray as well as CCD images we compute the following estimation error measurement: 


\begin{tabular}{|l||c|c|c|c|}
\hline \multicolumn{1}{|c||}{} & \multicolumn{2}{c|}{ angle of rotation (in deg.) } & \multicolumn{2}{c|}{ maximum rotation error (in deg.) } \\
\cline { 2 - 5 } & mean & std. dev. & mean & std. dev. \\
\hline X-ray & 0.012 & 0.010 & 0.083 & 0.060 \\
optical camera & 0.063 & 0.052 & 0.110 & 0.066 \\
\hline
\end{tabular}

Table 1. Error in estimation of angle of rotation and maximum rotational error using X-ray and optical projection matrices versus ground truth of motion platform

- maximum rotational error: angle of the residual rotation $\tilde{\mathbf{R}}^{T} \mathbf{R}$, between estimated rotation and the ground truth. This represents the maximum rotational error with regard to both estimation of angle of rotation and rotation axis

- angle of rotation: difference between the estimated angle of rotation and the ground truth given by the motion platform.

The errors in the estimation of translational motion are negligible. We therefore compare different approaches only based on their success in estimation of dominant rotational motion.

Figure 3 depicts the absolute error in estimation of angle of rotation for both X-ray and optical phantoms for the fist experiment. Both Figure 3, and table 1 show that accuracy of the estimated rotation is better for X-ray projection matrices. However, these figures also show that both methods determine the rotation with high accuracy. Both methods determine the rotation with enough precision needed for $3 \mathrm{D}$ reconstruction.

\subsection{Moving C-arm}

In this experiment the $\mathrm{C}$-arm is rotated 190 degrees around a reconstruction phantom (see Fig. 5(c)). Eighty five pairs of X-ray and optical images are taken during the $\mathrm{C}$-arm motion. Additionally the 3D pose information of a marker plate attached to the X-ray tube is measured by an external optical tracking system (Polaris from Northern Digital). The C-arm is not able to provide the motion parameters with acceptable accuracy. Therefore, experimental results are primarily validated and compared with regard to the quality of the 3D reconstruction process.

In addition we also want to compare the results achieved with the optical phantom and the external tracking system in a quantitative way. For both methods, we compare the estimated motion between two consecutive image frames with respect to the estimated motion using the X-ray phantom, see [7] for more detail. The result of our experiment with motion platform, see sect. 3.1, lets us assume that this ground truth motion is estimated quite accurately. Both X-ray and optical phantom are physically attached and defined such that the C-arm motion is described in a common coordinate system. This is not the case for the external tracking system. In order not to add errors due to the extra necessary calibration between the two coordinate systems, we only compare the results of 

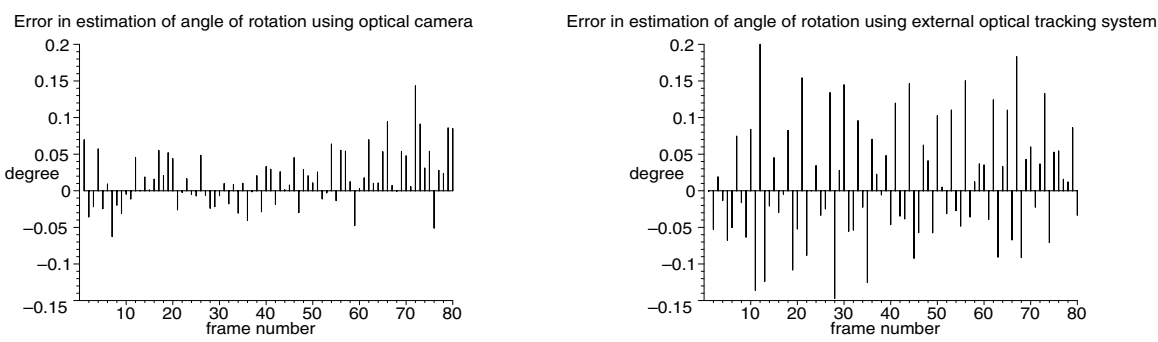

Fig. 4. Experimental results of estimation of C-arm motion: Error in estimation of angle of rotation using optical camera (left) and external tracking system (right) versus ground truth of X-ray projection matrices

estimating the angle of rotation, which is independent of the choice of coordinate system.

Figures 5(a-c) show the experimental setup. The left image shows the C-arm, $\mathrm{X}$-ray and optical phantom as well as the external tracking system. The closeup in the middle shows the CCD camera attached to the X-ray tube and the marker plate in front of the X-ray beam. The right image shows our reconstruction phantom. It consists of two cylindrical objects covered in an acrylic hull. Attached to it (not visualized in the picture) is a metal ring made from titanic alloy positioned inside an acrylic cover.

The X-ray and camera projection geometry are computed using an initial frame (in clinical applications this would be done off-line). The intrinsic geometry of this X-ray projection is used to define the virtual detector plane (see sect. 2.2).

Figures 5(d-f) show 3D reconstruction results using projection matrices obtained by the different calibration methods. These figures show the three orthogonal Maximum Intensity Projections (MIP) of the reconstructed volume. The differences are not dramatic and especially the amount of artifacts caused by the metallic part is very small for all three 3D reconstructions. Comparing the two new methods, the $3 \mathrm{D}$ reconstruction using the attached CCD camera matrices achieves better results than using the external tracking system. Figure 4 shows the absolute error in angle of rotation using CCD camera and external tracking system versus the assumed ground truth of X-ray projection matrices. The mean absolute error and standard deviation are smaller using optical projection matrices, see table 2. In average, the errors for this experiment are comparable to the errors of the experiment with the motion platform described in sect. 3.1. 


\begin{tabular}{|l||c|c|}
\hline rotation angle (in deg.) & mean & std. dev. \\
\hline optical camera & 0.031 & 0.027 \\
external tracking system & 0.065 & 0.047 \\
\hline
\end{tabular}

Table 2. Experimental results of estimation of C-arm motion: Error in estimation of angle of rotation using optical camera and external tracking system versus ground truth of X-ray projection matrices

Finally, Fig. 5(g) presents a 3D reconstruction of anatomical data. A part of a pig's spine has been reconstructed using our Camera-Augmented Mobile C-arm (CAMC). The reconstruction seems to be rich and precise enough to be used in interventional procedures. This must be fully tested and evaluated through clinical trials.

\section{Conclusion and Future Work}

In this paper, we propose to add optical cameras to a low-cost mobile C-arm. The optical system takes the responsibility of dynamically calibrating the C-arm motion during the patient run. The experimental results show that with this on-line calibration process one can recover the X-ray projection geometry and reconstruct the volume of interest. This integrated camera solution was compared to the use of an external tracking system for motion estimation and achieved better reconstruction result. Moreover, there is a need for on-line calibration of external sensor and X-ray imaging system which is not needed if the CCD camera is rigidly and permanently attached to the $\mathrm{C}$-arm. The mobile $\mathrm{C}$-arm is already being used in medical procedures. Therefore, Camera-Augmented Mobile C-arms have a great chance of getting easily accepted and used in clinical applications. The reconstruction results are quite satisfactory. Having shown the feasibility of our approach we will continue working on the design of a phantom that can be used in the clinical environment. This CAMC based reconstruction will reduce the need of pre-computation of the 3D geometry with another modality, and therefore reduces the complexity of on-line registration with X-ray fluoroscopic images. We also plan to use the optical images for real-time navigation and for real-time merging of patient's X-ray and optical images in the near future.

\section{Acknowledgement}

Authors would like to thank K. Wiesent and W. Seissler for providing the 3D reconstruction software. We would also like to thank A. Bani-Hashemi and R. Graumann for useful discussion during the progress of this work. This work was accomplished thanks to partial support from Bayerische Forschungsstiftung. 


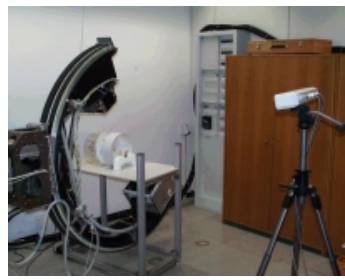

(a) Experimental setup

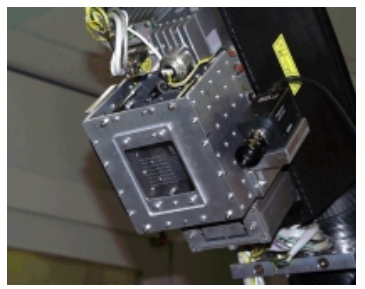

(b) Camera and marker plate

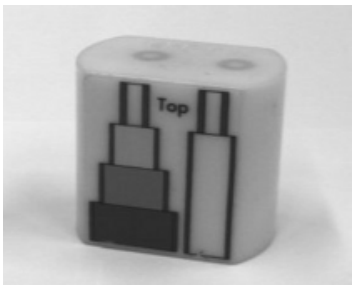

(c) Reconstr. phantom

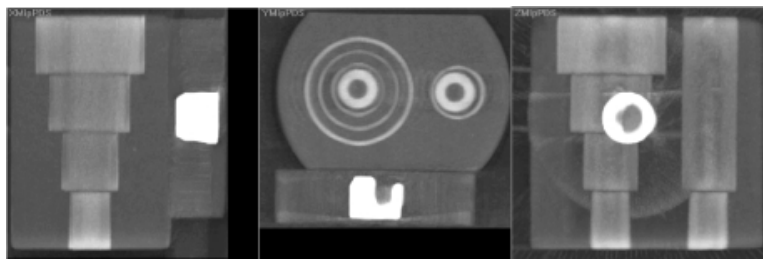

(d) 3D reconstruction using X-ray phantom

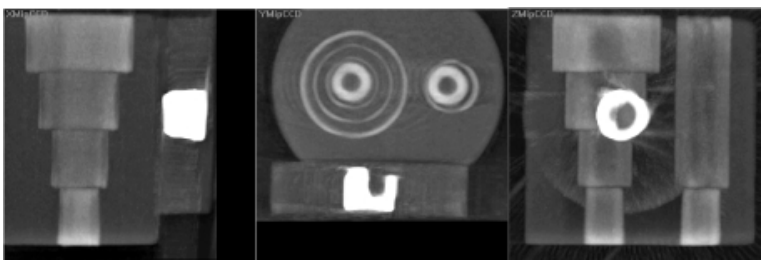

(e) 3D reconstruction using optical phantom

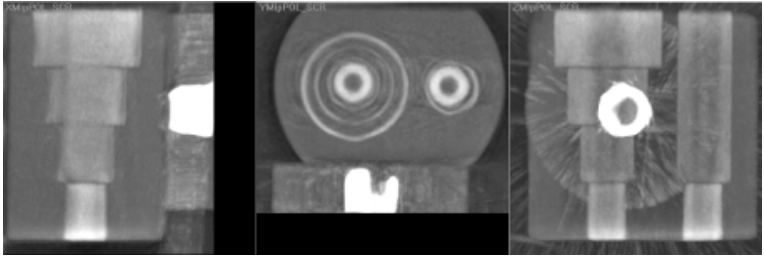

(f) 3D reconstruction using external tracking system

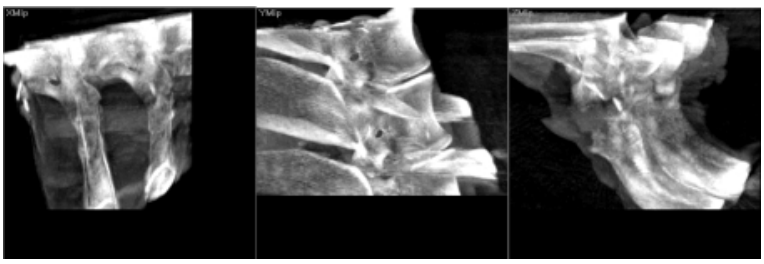

(g) 3D reconstruction of a part of a pig's spine

Fig. 5. Experimental setup (a,b), reconstruction phantom (c), and 3D reconstruction results $(d, e, f, g)$ 


\section{References}

1. M. Blackwell et al. An image overlay system for medical data visualization. In First International Conference on Medical Image Computing and Computer-Assisted Intervention (MICCAI), pages 232-240, 1998. 689

2. R. Koppe et al. 3d vessel reconstruction based on rotational angiography. In Computer Assitsted Radiology, pages 101-107. Springer, June 1995. 690

3. R. Fahrig, A. J. Fox, and D. W. Holdsworth. Characterization of a c-arm mounted xrii for 3d image reconstruction during interventional neuroradiology. pages 351360. Proc. SPIE 2708, 1996. 690

4. R. Fahrig, M. Moreau, and D. W. Holdsworth. Three-dimensional computer tomographic reconstruction using a c-arm mounted xrii: Correction of image intensifier distortion. Medical Physics, 24:1097-1106, 1997. 690

5. W. E. L. Grimson et al. Clinical experience with a high precision image-guided neurosurgery system. In MICCAI'98, pages 63-73, Cambridge, MA, USA, 1998. 689

6. E. Gronenschild. The accuracy and reproducibility of a global method to correct for geometric image distortion in the x-ray imaging chain. Medical Physics, 24:18751888, 1997. 690

7. N. Navab, A. Bani-Hashemi, M. S. Nadar, K. Wiesent, P. Durlak, T. Brunner, K. Barth, and R. Graumann. 3D reconstruction from projection matrices in a carm based 3d-angiography system. In MICCAI'98, pages 1305-1306, Cambridge, MA, USA, 1998. 690, 692, 693, 694

8. D. L. Parker. Optimal short scan convolution reconstruction for fanbeam ct. Medical Physics, 9(2):254-257, March/April 1982. 690

9. A. Rougèe, A. C. Picard, Y. Trousset, and C. Ponchut. Geometrical calibration of $\mathrm{x}$-ray imaging chains for three-dimensional reconstruction. Computerized medical Imaging and Graphics, 17(4/5):295-300, 1993. 690 